Revue internationale P.M.E.

Économie et gestion de la petite et moyenne entreprise

Revure

internationale

PME

\title{
Théories financières et endettement des PME en France Une analyse en panel
}

\section{Philippe Adair et Mohamed Adaskou}

Volume 24, numéro 3-4, 2011

URI : https://id.erudit.org/iderudit/1013665ar

DOI : https://doi.org/10.7202/1013665ar

Aller au sommaire du numéro

Éditeur(s)

Presses de l’Université du Québec

ISSN

0776-5436 (imprimé)

1918-9699 (numérique)

Découvrir la revue

Citer cet article

Adair, P. \& Adaskou, M. (2011). Théories financières et endettement des PME en France : une analyse en panel. Revue internationale P.M.E., 24(3-4), 137-171. https://doi.org/10.7202/1013665ar

\section{Résumé de l'article}

Nous présentons les hypothèses relatives aux deux principales théories financières de la structure d'endettement des PME - compromis et financement hiérarchique - en mettant l'accent sur les imperfections du financement : fiscalité, coûts d'agence et asymétrie d'information. Nous testons ces hypothèses, la variable expliquée étant le taux d'endettement total, grâce à un modèle linéaire de données de panel, sur un échantillon de 1520 PME françaises observées durant la période 2000-2004. Selon nos résultats, à l'exception des garanties qui conditionnent l'accès au crédit, la théorie du compromis est infirmée au regard de la relation entre l'endettement et la taille ainsi que l'âge des PME ; la relation entre l'endettement et le taux d'investissement ainsi que le crédit interentreprises confirme la théorie du financement hiérarchique. 


\title{
Théories financières et endettement des PME en France Une analyse en panel
}

\author{
Philippe ADAIR \\ Mohamed ADASKOU \\ ÉRUDITE, Université Paris-Est Créteil
}

\section{MOTS CLÉS}

\section{Données de panel - PME - Structure du capital - Théorie du compromis - Théorie du financement hiérarchique}

\begin{abstract}
LES AUTEURS
PHILIPPE ADAIR est maître de conférences en sciences économiques à la Faculté de sciences économiques et de gestion, Université Paris-Est Créteil, France, chercheur au laboratoire ÉRUDITE en analyse monétaire et financière (cycles, microfinance) et en économie du développement (économie informelle, segmentation du marché du travail, microentreprises). II a récemment publié dans des revues scientifiques telles que Mondes en développement Région et Développement et Revue économique. Adresse: ÉRUDITE, Faculté de sciences économiques et de gestion, Université Paris-Est Créteil, 61, avenue du Général-de-Gaulle, 94000 Créteil, France. Courriel: <adair@u-pec.fr>.

MOHAMED ADASKOU est maître assistant en sciences économiques à la Faculté de sciences économiques et de gestion, Université lbn-Zohr, Agadir, Maroc. Il est chercheur au laboratoire ÉRUDITE en analyse financière et PME. II a réalisé sa thèse en 2009 sous la direction de Philippe Adair. Adresse: ÉRUDITE, Faculté de sciences économiques et de gestion, Université Paris-Est Créteil, 61, avenue du Général-de-Gaulle, 94000 Créteil, France. Courriel: $<$ madaskou92@gmail.com>.
\end{abstract}

\section{RÉSUMÉ}

Nous présentons les hypothèses relatives aux deux principales théories financières de la structure d'endettement des PME - compromis et financement hiérarchique - en mettant l'accent sur les imperfections du financement: fiscalité, coûts d'agence et asymétrie d'information. Nous testons ces hypothèses, la variable expliquée étant le taux d'endettement total, grâce à un modèle linéaire de données de panel, sur un échantillon de 1520 PME françaises observées durant la période 
2000-2004. Selon nos résultats, à l'exception des garanties qui conditionnent l'accès au crédit, la théorie du compromis est infirmée au regard de la relation entre l'endettement et la taille ainsi que l'âge des PME; la relation entre l'endettement et le taux d'investissement ainsi que le crédit interentreprises confirme la théorie du financement hiérarchique.

\begin{abstract}
We present the assumptions for two main theories of the financial structure of SMEs debt financing, i.e. tradeoff theory and pecking order theory; with a focus on the imperfections of funding: taxation, agency costs and asymmetric information. We test these assumptions, the dependent variable being the total debt ratio, thanks to a linear panel data model applied upon a sample of 1520 French SMEs over 2000-2004. According to our results, except for the guarantees upon which access to credit is granted, tradeoff theory is reversed with respect to the relationship between debt, and the size and age of SMEs, whereas the relationship between debt and the investment rate, and trade credit support the pecking order theory.
\end{abstract}

\title{
RESUMEN
}

Se presentan los supuestos para los dos principales teorías sobre la estructura de la deuda financiera de las PyMEs, endeudamiento óptimo y financiación jerárquica - con especial atención a las imperfecciones del financiamiento: impuestos, costes de agencia y la información asimétrica. Ponemos a prueba estas hipótesis, la variable dependiente es la ratio de deuda total, gracias a un modelo lineal con datos de panel, una muestra de 1.520 pymes francesas observadas en el período 2000-2004. Según nuestros resultados, a excepción de las garantías sobre los cuales se concede el acceso al crédito, la teoría del endeudamiento óptimo se invierte con respecto a la relación entre la deuda y el tamaño y edad de las PyMEs, mientras que la relación entre la deuda y la tasa de inversión y el crédito comercial apoyan la teoría de la financiación jerárquica.

\section{ZUSAMMENFASSUNG}

In Anlehnung an die zwei massgeblichen Theorien zur Finanzierung von KMU Kompromiss und hierarchische Finanzierung - präsentieren wir die Hypothesen in Bezug auf die Unvollkommenheit der Finanzierung: Steuern, Agenturkosten und asymmetrische Information. Die Hypothesen mit der Schuldenquote werden anhand von Paneldaten aus den Jahren 2000 bis 2004 von 1520 französischen KMU mittels eines linearen Modelles getestet. Mit Ausnahme von Garantien, die den Zugang zu Krediten ermöglichen, zeigen die Resultate auf, dass die Kompromisstheorie die Beziehung zwischen Verschuldung und der Grösse sowie des Alters der KMU entkräften. Die Beziehung zwischen Verschuldung, Investitionsquote sowie zwischenbetrieblichen Krediten bestätigen die Theorie der hierarchischen Finanzierung. 


\section{Introduction}

Les petites et moyennes entreprises (PME) occupent une place importante dans le tissu économique en France où les microentreprises ou TPE (0-9 salariés) représentent $92,85 \%$, contre $6 \%$ pour les petites entreprises (10-49 salariés) et seulement $1 \%$ pour les moyennes entreprises (50-249 salariés). Les PME constituent le principal pourvoyeur d'emplois (60\% des emplois) et contribuent à $55 \%$ de la valeur ajoutée (Savajol, 2003).

Cette répartition est quasi identique à celle des 20,5 millions d'entreprises en Europe : soit, selon l'Observatoire européen des PME (2003), près de $93 \%$ de microentreprises, $6 \%$ de petites entreprises, moins de $1 \%$ de moyennes entreprises et seulement $0,2 \%$ de grandes entreprises (plus de 250 salariés).

Ces entreprises éprouvent des difficultés pour financer leurs projets d'investissement (Coluzzi, Ferrando et Martinez-Carrascal, 2009; Savignac et Sevestre, 2008). Cela tient notamment à leur capacité limitée à fournir une information fiable ainsi qu'à leur expertise financière limitée qui résulte de leurs spécificités financières. Selon Ang (1992), ces spécificités sont liées à la difficulté de séparer les ressources financières de l'entreprise de celles de son propriétaire, aux problèmes de succession qui peuvent rendre le développement de l'entreprise incertain, à l'insuffisance ou à «l'opacité informationnelle» de leur système d'information interne et externe, à l'importance des relations informelles et à la présence de contrats implicites, etc. La fragilité financière d'une PME peut raccourcir son cycle de vie, une mauvaise conjoncture engendrant la défaillance qui résulte aussi d'un risque d'erreurs plus important en raison d'un manque d'expérience, d'un manque d'expertise ou simplement d'un optimisme exagéré de l'entrepreneur.

Il convient d'expliquer pourquoi les PME se plient mal aux hypothèses conventionnelles du modèle réduit de la grande entreprise (Belletante, Levratto et Paranque, 2001).

Plusieurs synthèses des travaux théoriques et empiriques ont récemment été consacrées aux PME dans les pays développés, notamment en Europe (Balling, Bernet et Gnan, 2009) et singulièrement en France (Chertok, De Malleray et Pouletty, 2009). Ces travaux mettent principalement l'accent sur le financement - interne ou externe - des PME et sur la pertinence des théories concurrentes au regard de l'explication de leur structure de financement.

Notre étude s'inscrit dans ce contexte théorique et empirique afin d'examiner les principaux déterminants du taux d'endettement des PME et d'expliquer l'incidence des caractéristiques de ces entreprises sur leur structure 
financière. Nous répondons aux questions suivantes: les théories financières, essentiellement destinées aux grandes entreprises, expliquent-elles les choix de financement des PME? Ces entreprises privilégient-elles un taux d'endettement optimal ou bien une hiérarchie des sources de financement?

Si nombre d'études portent sur la structure financière des grandes entreprises cotées, nous mettons l'accent sur les PME. L'analyse d'un échantillon de 1520 PME françaises (non cotées) observées sur une période relativement récente (2000-2004) nous permet de comparer nos résultats avec ceux d'autres travaux antérieurs ou contemporains relatifs aux PME, notamment en France ( $c f$. annexe IV).

Notre étude est organisée comme suit: la section 1 examine la contribution des théories financières, essentiellement la théorie du compromis et celle du financement hiérarchique, à l'explication du choix de financement des PME; la section 2 présente la grille des hypothèses empiriques qui visent à tester les deux principales théories; la section 3 décrit les caractéristiques de l'échantillon; la section 4 expose le modèle économétrique utilisé et en interprète les résultats empiriques au regard de ceux des travaux français. Cette dernière section est suivie d'une conclusion.

\section{Les théories de la structure du capital et les PME}

La théorie de la structure du capital remonte à l'article de Modigliani et Miller (1958), qui démontrent, sous certaines hypothèses, que les marchés de capitaux sont parfaits, qu'il n'y a pas de taxes ni de coûts d'agence ou de coûts de transaction, et que la structure financière est neutre par rapport à la valeur de l'entreprise. La suite de la littérature insiste beaucoup sur l'assouplissement de ces hypothèses de neutralité de la structure financière afin de rapprocher la théorie de la réalité des entreprises.

L'état actuel de l'analyse de la structure du capital recouvre une grande variété d'approches, mais il n'existe pas une théorie universelle concernant le choix entre fonds propres et dettes, voire le recours au marché financier (Myers, 2001; Harris et Raviv, 1991). Les différentes théories de la structure optimale du capital dépendent des conditions économiques et des particularités financières de la firme (Daskalakis et Psillaki, 2007).

Nous distinguons deux principales approches de la structure financière. La théorie du compromis ou trade-off theory (TOT) suppose que la structure optimale est atteinte selon un arbitrage entre les avantages et les inconvénients des principales sources de financement. La théorie du financement hiérarchique ou pecking order theory (POT), contrairement à la précédente, 
récuse la détermination d'un taux d'endettement optimal (ratio cible), mais privilégie l'existence d'une hiérarchie des sources de financement établie sur l'hypothèse d'asymétrie d'information.

\subsection{La théorie du compromis}

La théorie du compromis procède de la révision du théorème de Modigliani et Miller (1958) au regard de la fiscalité et des coûts de faillite, puis de l'inclusion de nouvelles hypothèses - coûts d'agence et valeur du signal - qui viennent enrichir la procédure d'optimisation (l'atteinte d'un ratio cible) et conduisent à dépasser le cadre statique de l'équilibre pour l'inscrire dans une perspective dynamique. Nous présentons brièvement ces diverses hypothèses qui concernent essentiellement les grandes entreprises et les entreprises cotées, et dont nous examinons la pertinence au regard des particularités des PME.

Modigliani et Miller (1963) prennent en considération la fiscalité, notamment la déductibilité des intérêts financiers sur le résultat imposable, et montrent que la valeur de la firme endettée est égale à la valeur de la firme non endettée, augmentée de l'économie d'impôt réalisée grâce à l'endettement. Ainsi, la déductibilité des intérêts inciterait les entreprises à recourir à l'endettement plutôt qu'aux fonds propres, dont les rémunérations ne sont pas déductibles. Par conséquent, la valeur d'une entreprise endettée est supérieure à celle d'une entreprise sans dette à la condition que les économies d'impôt ne bénéficient qu'à l'entreprise et ne concernent pas les revenus des particuliers (Miller, 1977).

Concernant les PME, le taux d'imposition réduit dont bénéficient ces entreprises limite, jusqu'à un certain point, la pratique d'une politique d'endettement conditionnée à la déductibilité des charges d'intérêt (Ang, 1991) ${ }^{1}$. De plus, la réalisation des économies d'impôt liées à l'endettement est influencée par la variabilité supérieure des investissements et le caractère plus incertain des bénéfices réalisés par ce type d'entreprises (Pettit et Singer, 1985).

1. Afin de réduire le coût du financement et améliorer les capitaux propres des TPE et PME indépendantes, en 1996 puis en 2001, leur charge fiscale a été allégée en instaurant un taux réduit d'impôt sur les sociétés (15\% au lieu du taux normal de $33,33 \%$ ) sur une fraction de leur bénéfice fiscal plafonné à $38120 €$. Sur environ un million d'entreprises assujetties en 2004, une sur deux bénéficie effectivement du taux réduit, essentiellement des microentreprises et des petites entreprises (moins de 50 salariés) ayant moins de sept ans d'activité. Parmi ces entreprises, $96 \%$ ont un chiffre d'affaires inférieur à 7,63 M€ et, pour $80 \%$ d'entre elles, il est inférieur à $1 \mathrm{M} €$ (Raspiller, 2007). 
L'existence des coûts de faillite met en cause la relation de proportionnalité entre la valeur de l'entreprise et l'avantage fiscal (Stiglitz, 1969). La confrontation des bénéfices fiscaux et des coûts de faillite doit théoriquement aboutir à la détermination d'un niveau d'endettement optimal qui est atteint lorsque les bénéfices marginaux liés à la déductibilité des charges financières égalisent les coûts marginaux de faillite liés à l'augmentation de l'endettement.

De même, l'introduction de coûts d'agence permet de déterminer une structure optimale de capital (Jensen et Meckling, 1976). Les intérêts des dirigeants de l'entreprise (mandants) ne coïncident pas parfaitement avec ceux de ses partenaires (mandataires) et engendrent inévitablement des coûts d'agence qui affectent le financement de l'entreprise. Ces coûts d'agence sont de trois types: les coûts de contrôle ou de surveillance, les coûts de limitation et les coûts d'opportunité. Les conflits d'intérêts entre les actionnaires et les créanciers résultent du fait qu'en cas de faillite, les créanciers ont priorité sur les actionnaires. Un endettement optimal est atteint lorsque les coûts d'agence sont minimaux. Concernant les PME, il n'y a pas ou très peu de coûts d'agence entre les dirigeants et actionnaires parce que dans ces entreprises, les dirigeants sont probablement les propriétaires. Cependant, les conflits d'agence entre les propriétaires et les prêteurs sont importants. Adam, Farber et Michel (1989) de même que Colot et Michel (1996) affirment que le banquier ou le fournisseur de capital-risque (le principal) contrôlent difficilement les agissements du dirigeant de la petite firme (l'agent), surtout par manque de transparence des moyens d'information, et que cette situation entraîne la croissance des coûts d'agence.

Le financement bancaire est la source de financement par emprunt la plus utilisée par les entrepreneurs, surtout en ce qui concerne le crédit à court terme, et ce, dans la plupart des pays industrialisés (St-Pierre,1999). Toutefois, la relation banques-PME est caractérisée par l'existence d'une méfiance réciproque. Les banques jugent souvent les projets présentés par les PME plus risqués que ceux des grandes entreprises. Les PME estiment que les banques surévaluent le risque de leurs projets et leur imposent des primes de risque trop élevées qui renchérissent le coût du crédit (Dell'Ariccia et Marquez, 2004). Si les conflits d'agence interviennent entre les PME et les banques, celles-ci ne prêtent que si les conséquences des asymétries d'information sont levées; sinon, l'équilibre du marché du crédit engendre un rationnement tel que, parmi les entreprises a priori identiques, certaines obtiennent un crédit alors que d'autres n'en reçoivent pas même si elles sont prêtes à payer un prix plus élevé (Stiglitz et Weiss, 1981). Stiglitz et Weiss (1981) avancent la notion de sélection adverse, suivant laquelle plus les rendements escomptés d'un projet d'investissement sont élevés, plus celui-ci est risqué. Des forts taux 
débiteurs peuvent donc favoriser l'adoption de comportements téméraires de la part des emprunteurs et exercer une influence négative sur les recettes anticipées des banquiers. La fixation d'un coût du crédit tel que les offres et demandes s'égalisent ne constitue pas un comportement rationnel de la part des institutions financières (Levratto, 1992). Cependant, les banques peuvent notamment réduire les conséquences des asymétries d'information et inciter les entreprises à rembourser leur dette à l'échéance (Bruns et Fletcher, 2008) en leur proposant des contrats révélateurs qui consistent à établir le degré de risque de l'emprunteur potentiel au cours du temps; ces contrats correspondent à des combinaisons diverses entre le taux d'intérêt et le montant des garanties, visant à construire une relation de long terme (Psillaki, 1998).

La théorie du signal peut également expliquer le poids de l'endettement dans le passif de certaines entreprises. Cette théorie suppose que les agents internes à l'entreprise, plus informés que les agents externes que sont principalement les banques dans le cas des PME, ont intérêt à transmettre une partie de l'information à ces derniers par l'intermédiaire d'un signal afin d'accéder à la dette. Ross (1977) préconise une solution de signalisation par le niveau de l'endettement de la firme: l'entreprise qui a un niveau d'endettement plus élevé est présumée être de bonne qualité, et entreprend des projets d'investissement rentables. Lelet et Pyle (1977) préconisent une solution de signalisation reposant sur le degré d'implication du dirigeant lui-même dans le financement des projets d'investissement; cette implication révèle que le dirigeant est un bon manager et, en conséquence, les créanciers ont la conviction que le projet envisagé par ce type de dirigeant est rentable sinon ils n'auraient pas dû engager leurs fonds.

Il convient enfin d'ajouter que le ratio cible d'endettement optimal procède non d'un ajustement instantané, correspondant à la théorie statique du compromis, mais d'une trajectoire qui caractérise la théorie dynamique du compromis et conduit celle-ci à incorporer certains aspects de la théorie du financement hiérarchique (Dufour et Molay, 2010).

\subsection{La théorie du financement hiérarchique et les PME}

La théorie du financement hiérarchique, ou pecking order theory (POT), développée par Myers et Majluf (1984), est fondée sur l'asymétrie d'information qui existe entre les acteurs internes de l'entreprise (propriétaires, dirigeants) et ses acteurs externes (bailleurs de fonds). Les dirigeants adoptent une politique financière qui a pour but de minimiser les coûts associés à l'asymétrie d'information et ils préfèrent le financement interne au financement externe. Selon cette théorie, le dirigeant hiérarchise ses préférences selon la séquence suivante: l'autofinancement, la dette non risquée, la dette risquée, 
l'augmentation du capital. Le respect de cette hiérarchie a pour avantages d'éviter la réduction des prix des actions de l'entreprise, de limiter la distribution des dividendes pour augmenter l'autofinancement, de réduire le coût du capital en limitant le plus possible le recours aux emprunts. Les entreprises rentables ont donc plus de financement interne disponible.

Ang (1991) souligne que cette théorie peut être facilement appliquée au cas des PME, lesquelles n'ont pas pour objectif de réaliser une structure financière optimale, mais dont les décisions de financement visent à ordonner leurs préférences pour les financements internes par rapport aux financements externes et pour la dette par rapport aux fonds propres. Nombre de PME souhaitent emprunter quand les besoins de financement de leur investissement dépassent les flux de trésorerie générés en interne, mais font souvent face dans leur relation de crédit à la sélection adverse et à des coûts d'information. Ces coûts peuvent être nuls pour les fonds internes (autofinancement), mais sont élevés dans le cas de l'émission de nouvelles actions, faisant que les coûts de la dette se trouvent dans une position intermédiaire. L'objectif des dirigeants de PME est de maximiser leur propre richesse tout en conservant leur indépendance vis-à-vis des acteurs externes; c'est pourquoi les fonds internes font l'objet en premier lieu de leur choix de financement. Si les fonds internes sont insuffisants, ils préfèrent recourir à la dette plutôt qu'à l'augmentation du capital, car la dette a l'avantage de réduire le degré de dépendance de l'entreprise à l'égard des autres apporteurs de capitaux, ce qui leur permet de garder le contrôle et le pouvoir de décision.

La définition du financement hiérarchique fondé sur la séquence fonds propres - dette non risquée - dette risquée - ouverture du capital est discutée par Vasiliou, Eriotis et Daskalakis (2009); ils signalent que la priorité à la mobilisation des ressources internes, si elle est avérée, n'implique pas nécessairement que l'étape suivante corresponde à la dette. Il y aurait lieu de dissocier une étape initiale de la POT (fonds propres) d'une seconde étape de la POT (dette ou ouverture du capital).

La POT a été argumentée au regard d'hypothèses relatives à l'âge et à la profitabilité des entreprises. À l'appui de la POT, Berger et Udell (1990) font valoir que les PME recourent moins à l'endettement au fur et à mesure que leur cycle de vie les conduit de la jeunesse à la maturité. Bulan et Yan (2010) considèrent de même que la maturité permettrait aux entreprises d'accéder aux marchés. Vanecker et Manigart (2010) suggèrent une «POT étendue» qui distingue entreprises profitables (POT 1) et entreprises non profitables (POT 2) et qui peut s'appliquer aux entreprises à potentiel élevé de croissance. Une version élargie de la POT tiendrait compte de la propriété 
du capital en mettant l'accent sur l'appartenance des PME à un groupe, qui permet d'accroître la capacité à mobiliser des ressources internes. Nous n'avons pas considéré ici le critère d'indépendance des $\mathrm{PME}^{2}$.

\section{La grille des hypothèses}

L'ensemble des travaux précédemment évoqués inspire en partie les hypothèses retenues dans notre étude dont nous présentons la grille.

La variable expliquée est le taux d'endettement de l'entreprise (TD) qui est mesuré par l'importance des dettes dans le total du passif, c'est-à-dire par le rapport des dettes, sans distinction de nature et d'échéance (court terme et moyen long terme), au total du bilan. Ce ratio reflète le choix entre capitaux propres et dettes; il est utilisé comme variable expliquée de la structure financière par Bourdieu et Colin-Sédillot (1993) et par Biais, Hillion et Malécot (1995).

Les variables explicatives sont choisies de façon à tenir compte des particularités financières des PME. Nous avons retenu des indicateurs prenant en considération simultanément des contraintes d'accès aux ressources et des effets directement liés à l'asymétrie d'information et aux conflits d'agence: taille, âge, secteur, taux d'investissement, crédit interentreprises, garanties et marge d'autofinancement. Eu égard aux difficultés relatives à l'obtention d'information, nous n'introduisons pas de variables concernant les effets de la fiscalité.

La taille (EFF), correspondant à l'effectif salarié3, est introduite grâce à une variable indicatrice muette ou dummy. Sans aucun doute, la taille joue un rôle déterminant dans le choix entre action et dette. Les grandes entreprises sont moins exposées au risque de faillite que leurs homologues de petite taille du fait qu'elles diversifient leurs investissements; par conséquent, la taille doit être positivement liée au levier financier. En outre, les grandes entreprises peuvent être en mesure d'engager des coûts de transactions plus faibles associés à la dette. Enfin, les coûts d'information sont plus faibles

2. Selon Kremp et Philippon (2008), la proportion des PME indépendantes diminue rapidement entre 1997 et 2006: de 4 sur 5 à 1 sur 2 au cours de la période.

3. Nous n'avons pas retenu le montant du chiffre d'affaires (CA) ou du bilan, dont les seuils déterminent la classification des entreprises ( $c f$. annexe I). Tenir compte des trois critères à la fois peut surdéterminer l'échantillonnage ou a contrario le réduire, si les critères sont mutuellement exclusifs. Par ailleurs, le critère de l'effectif, quoique réel et non financier, est robuste et permet de comparer nos résultats avec les données issues d'autres sources (le fichier ALISSE de l'INSEE). 
pour les grandes entreprises en raison de la bonne qualité de l'information financière. Cependant, Rajan et Zingales (1995) ont observé que l'effet de taille est ambigu: la taille peut également être un indicateur d'information aux investisseurs externes, ce qui devrait augmenter la part des fonds propres plutôt que la part de la dette. Ainsi, les PME qui décident d'accroître leur effectif salarié peuvent apparaître aux yeux des investisseurs potentiels comme des entreprises qui ont des opportunités de croissance et qui sont en «bonne santé» financière, c'est-à-dire comme des PME qui sont moins exposées au risque de faillite, ce qui devrait augmenter leurs fonds propres plutôt que leur dette. Notre première hypothèse (H1) vise donc à tester l'apport de la théorie du compromis (TOT) dans l'explication de l'endettement des PME. Elle est formulée comme suit: la taille de l'entreprise devrait être liée positivement au niveau du taux d'endettement.

L'âge (AGE) correspond à la différence entre la première année d'observation (2000) et la date de création de l'entreprise: plus celle-ci est âgée, plus son capital informationnel est important. Selon la théorie du financement hiérarchique, et en supposant que la capacité d'autofinancement de la petite entreprise est une fonction croissante de son âge, nous pouvons inférer que les entreprises plus âgées ont recours de façon moins intensive à l'endettement. Inversement, la théorie du compromis (coûts de faillite et théorie de l'agence) présuppose l'existence d'une relation positive entre l'âge de l'entreprise et le taux d'endettement, du fait que les entreprises plus âgées ont une meilleure réputation et une plus grande expérience qui peuvent conduire à la réduction des coûts d'agence en raison d'un signal positif sur la bonne qualité des investissements potentiels. Notre deuxième hypothèse $(\mathrm{H} 2)$ vise donc à tester cette dernière théorie, celle du compromis (TOT); elle est formulée ainsi: les entreprises plus âgées ont un taux d'endettement plus élevé.

Le secteur d'activité (SEC) a un impact sur la structure financière de l'entreprise du fait que chaque secteur est caractérisé par des modes de fonctionnement propres et constitue un indicateur synthétique du risque lié à l'activité principale de l'entreprise (Psillaki, Tsolas et Margaritis, 2010). Cette variable est mesurée au moyen de quatre indicatrices sectorielles (dummy industrie, dummy commerce, dummy construction et dummy services) en utilisant la nomenclature d'activité française (NAF), niveau 60. Notre troisième hypothèse $(\mathrm{H} 3)$ peut être formulée comme suit:le secteur influence le taux d'endettement de l'entreprise.

Le taux d'investissement (TINV) ou plutôt sa croissance est susceptible de peser sur la capacité d'autofinancement et de conduire l'entreprise à emprunter. Myers (1977) fait valoir que les entreprises ayant un potentiel de croissance tendent à avoir un faible taux d'endettement : d'après la théorie de l'agence, les opportunités de croissance peuvent induire de l'aléa moral 
qui conduit les entreprises à prendre plus de risques; les entreprises qui ont un potentiel de croissance sont donc considérées comme risquées et ont du mal à convaincre leurs bailleurs de fonds de leur accorder des crédits. Une forte croissance devrait se traduire par la réduction sensible de l'endettement surtout pour la petite entreprise.

Dans le même sens, la théorie du financement hiérarchique affirme que l'entreprise en croissance soutenue se caractérise par un besoin de financement externe élevé, et l'endettement apparaît comme la source de financement la plus appropriée (Ziane, 2004). La combinaison d'une croissance forte et d'un accès restrictif aux marchés financiers devrait obliger les PME à solliciter d'une manière intensive le financement bancaire (Chittenden, Hall et Hutchinson, 1996).

L'effet de la croissance sur l'endettement s'avère donc ambigu. Si l'on se réfère aux enseignements de la théorie de l'agence, il devrait y avoir une relation négative entre ces deux variables. En revanche, si l'on se réfère aux prédictions de la théorie de financement hiérarchique, une relation positive est attendue. L'estimation de la croissance diffère d'une étude à l'autre: Chittenden et al. (1996) utilisent le taux de croissance de toute la période; nous nous plaçons dans la lignée de Bourdieu et Colin-Sédillot (1993), qui utilisent le taux d'investissement calculé en rapportant les immobilisations corporelles à la valeur ajoutée. Notre quatrième hypothèse $(\mathrm{H} 4)$ vise donc à tester les prédictions de la théorie du financement hiérarchique (POT) et est formulée ainsi: il devrait y avoir une relation positive entre le taux d'investissement et le taux d'endettement.

Le crédit interentreprises (CIE) résulte de la différence entre les montants bruts du crédit client et de la dette fournisseur. Pour disposer d'un outil de comparaison entre les différentes entreprises de notre panel, nous exprimons ce montant en pourcentage du bilan, c'est-à-dire que nous divisons le montant du solde du crédit interentreprises par le total du bilan. L'entreprise deviendra prêteuse ou emprunteuse selon le signe du solde de ses créances commerciales et de ses dettes commerciales. Par exemple, si les créances d'une entreprise sont supérieures à ses dettes, elle est prêteuse et assume donc une charge à financer d'un montant égal au solde. En revanche, si les créances sont inférieures aux dettes, l'entreprise est emprunteuse et le solde correspond à un emprunt auprès de ses fournisseurs et auprès de ses clients, sous forme d'avances sur commandes, destinées à financer son cycle d'exploitation. La capacité de l'entreprise à s'endetter auprès de ses partenaires commerciaux peut être perçue par les banques comme le signal d'une bonne fiabilité dans le paiement, ce qui contribue à augmenter l'endettement bancaire. Cependant, Petersen et Rajan (1994) considèrent que la dette commerciale est un substitut à la dette financière dans les entreprises rationnées. 
Selon l'Observatoire européen des PME (2001), pour un nombre considérable d'entreprises en phase de démarrage (voire en création), les crédits fournisseurs représentent la source la plus importante de leur fonds de roulement comparativement aux prêts bancaires. La relation entre la dette commerciale et la dette financière serait donc négative. Notre cinquième hypothèse (H5) vise donc à tester la prédiction de la théorie de financement hiérarchique (POT), le crédit interentreprises étant une source de financement à court terme importante et moins risquée pour les PME. Elle est formulée ainsi: le ratio du crédit interentreprises influence négativement le taux d'endettement.

L'exigence de garanties est le meilleur moyen utilisé par les bailleurs de fonds pour pallier le risque tout en constituant un moyen d'autosélection des emprunteurs. Il est peu probable que les intermédiaires financiers accordent des crédits, notamment à long terme, sans obtenir des garanties. Cet engagement peut dissuader les dirigeants de sous-investir et de faire des prélèvements discrétionnaires (Jensen et Meckling, 1976; Myers, 1977). L'exigence des garanties peut réduire les risques d'aléas de moralité:les garanties, sous forme d'éléments d'actif de l'entreprise ou d'apports personnels du dirigeant (Voordeckers et Steijvers, 2006), augmentent les pertes de l'entreprise et de l'entrepreneur en cas de défaut; c'est la raison pour laquelle l'entreprise est incitée a priori à entreprendre des projets moins risqués (Besanko et Thakor, 1987; Berger et Udell, 1990). Titman et Wessels (1988) soutiennent que les entreprises disposant d'éléments d'actif qui peuvent être utilisés comme garanties sont plus endettées. Cette thèse d'une relation inverse entre risques et garanties est rejetée par Stiglitz et Weiss $(1981,1987)$, qui affirment que l'exigence des garanties les plus élevées peut inciter l'emprunteur à entreprendre des projets plus risqués pour compenser le coût d'opportunité occasionné par la «mise en otage» des actifs collatéraux de l'entreprise. L'exigence de garanties élevées écarte également les agents les moins risqués qui présentent une faible aversion pour le risque. L'augmentation des garanties requises peut donc occasionner le développement d'un phénomène de sélection adverse.

La thèse de l'incapacité des garanties à révéler (ou à signaler) la qualité de la firme, défendue par Stiglitz et Weiss (1981), a été contestée par Bester (1985, 1987), qui démontre que l'apport d'éléments d'actif en garantie peut révéler la qualité de la firme. L'emprunteur à haut risque choisit un contrat dans lequel l'apport en garantie est faible et le taux d'intérêt élevé. En revanche,l'emprunteur à faible risque préfère un contrat doté d'un faible taux d'intérêt et d'un montant important de garantie. Ainsi, les contrats révélateurs, construits sur des combinaisons entre le taux d'intérêt et le montant des garanties, peuvent se substituer à la construction d'une information coûteuse sur l'entreprise. L'importance des garanties (GAR) de l'entreprise peut être déterminée à partir du poids des immobilisations corporelles et des stocks dans le bilan (Titman 
et Wessels, 1988; Bourdieu et Colin-Sédillot, 1993). Notre sixième hypothèse (H6) vise donc à tester la théorie du compromis (TOT); elle est formulée ainsi: les entreprises dont le total des immobilisations corporelles et des stocks est plus important dans le bilan ont un taux d'endettement plus élevé.

La marge d'autofinancement (MAF) est un indicateur des ressources de financement interne dont dispose l'entreprise qui peut être calculée en rapportant la capacité d'autofinancement à la valeur ajoutée (Bédué,1997). Cette variable peut refléter la pertinence de la théorie du financement hiérarchique dans le cas des PME. L'ensemble des travaux qui ont testé cette théorie affirme que ces entreprises préfèrent recourir en priorité au financement interne et ne recourent aux ressources externes qu'une fois que leur capacité d'autofinancement est épuisée. Notre septième hypothèse $(\mathrm{H} 7)$ vise à tester la théorie du financement hiérarchique (POT) : le taux d'endettement est inversement relié à la marge d'autofinancement.

Il existe plusieurs déterminants de la structure financière des entreprises qui diffèrent d'une étude à l'autre selon la théorie considérée. Le tableau 1 ci-dessous présente nos différentes variables et hypothèses, ainsi que les relations supposées entre la variable expliquée et les variables explicatives. Ces hypothèses seront testées empiriquement dans la section 5 afin de confronter les enseignements des différentes théories existantes au cas des PME.

TABLEAU 1

Récapitulatif des hypothèses testées

\begin{tabular}{|c|c|c|c|c|c|}
\hline $\begin{array}{l}\text { Théorie } \\
\text { financière }\end{array}$ & Hypothèse & $\begin{array}{l}\text { Variables } \\
\text { reflétant }\end{array}$ & $\begin{array}{c}\text { Mode } \\
\text { de calcul }\end{array}$ & Code & TD \\
\hline $\begin{array}{l}\text { Compromis } \\
\text { (TOT) }\end{array}$ & H1 & $\begin{array}{l}\text { Le choix entre } \\
\text { action et dette. }\end{array}$ & $\begin{array}{l}\text { Effectif salarié } \\
\text { moyen. }\end{array}$ & $\mathrm{EFF}$ & + \\
\hline $\begin{array}{l}\text { Compromis } \\
\text { (TOT) }\end{array}$ & $\mathrm{H} 2$ & $\begin{array}{l}\text { L'existence d'une } \\
\text { structure financière } \\
\text { optimale dans } \\
\text { une perspective } \\
\text { dynamique. }\end{array}$ & $\begin{array}{l}2000 \text { - date } \\
\text { de création. }\end{array}$ & $\mathrm{AGE}$ & + \\
\hline $\begin{array}{l}\text { Compromis } \\
\text { (TOT) ou } \\
\text { financement } \\
\text { hiérarchique } \\
\text { (POT) }\end{array}$ & H3 & $\begin{array}{l}\text { Le risque lié à } \\
\text { l'activité principale } \\
\text { de l'entreprise. }\end{array}$ & $\begin{array}{l}\text { Appartenance } \\
\text { au code NAF. }\end{array}$ & SEC & $\begin{array}{c}+\mathrm{ou} \\
-\end{array}$ \\
\hline $\begin{array}{l}\text { Financement } \\
\text { hiérarchique } \\
\text { (POT) }\end{array}$ & $\mathrm{H} 4$ & $\begin{array}{l}\text { Les opportunités } \\
\text { de croissance. }\end{array}$ & $\begin{array}{l}\text { Immobilisations } \\
\text { corporelles/ } \\
\text { valeur ajoutée. }\end{array}$ & TINV & + \\
\hline
\end{tabular}




\begin{tabular}{|c|c|c|c|c|c|}
\hline $\begin{array}{l}\text { Théorie } \\
\text { financière }\end{array}$ & Hypothèse & $\begin{array}{l}\text { Variables } \\
\text { reflétant }\end{array}$ & $\begin{array}{c}\text { Mode } \\
\text { de calcul }\end{array}$ & Code & TD \\
\hline $\begin{array}{l}\text { Financement } \\
\text { hiérarchique } \\
\text { (POT) }\end{array}$ & $\mathrm{H} 5$ & $\begin{array}{l}\text { Le crédit } \\
\text { fournisseur } \\
\text { est un substitut } \\
\text { à la dette } \\
\text { bancaire à } \\
\text { court terme. }\end{array}$ & $\begin{array}{l}\text { Crédit } \\
\text { clients-dettes } \\
\text { fournisseurs/ } \\
\text { total passif. }\end{array}$ & CIE & - \\
\hline $\begin{array}{l}\text { Compromis } \\
\text { (TOT) }\end{array}$ & H6 & $\begin{array}{l}\text { L'exigence } \\
\text { des garanties } \\
\text { et les risques } \\
\text { d'aléa moral. }\end{array}$ & $\begin{array}{l}\text { Immobilisation } \\
\text { corporelle } \\
\text { +stocks/total } \\
\text { actif. }\end{array}$ & GAR & + \\
\hline $\begin{array}{l}\text { Financement } \\
\text { hiérarchique } \\
\text { (POT) }\end{array}$ & $\mathrm{H} 7$ & $\begin{array}{l}\text { Les ressources } \\
\text { internes dont } \\
\text { dispose } \\
\text { l'entreprise. }\end{array}$ & $\begin{array}{l}\text { Capacité } \\
\text { d'auto- } \\
\text { financement/ } \\
\text { valeur ajoutée. }\end{array}$ & MAF & - \\
\hline
\end{tabular}

\section{Source des données et statistiques descriptives}

\subsection{Construction de l'échantillon}

Les données que nous utilisons sont issues de la base de données DIANE (Disque pour l'Analyse économique), qui contient les informations comptables, bilans et comptes de résultats, de 1085000 entreprises françaises. Elle contient également de nombreux renseignements relatifs à l'activité, l'actionnariat, les participations, les administrateurs, certains éléments des statuts (date de création, forme juridique, etc.), ainsi qu'une série de 50 ratios précalculés. En plus de la facilité d'accès, la disponibilité des données sur une période de plusieurs années ainsi que la richesse des informations obtenues favorisent le choix de cette source d'information ${ }^{4}$.

La sélection de l'échantillon des PME dans cette base de données nécessite tout d'abord une définition des PME qui diffère d'un pays à l'autre et d'un organisme à l'autre. Dans cette étude, nous retenons la dernière définition de la Commission européenne en 2003, qui considère la PME comme toute entreprise qui emploie moins de 250 personnes ( $c f$. annexe I).

4. Celle-ci a largement été utilisée dans d'autres études antérieures sur des panels cylindrés: 1387 firmes de 1989 à 1993 (Bédué, 1997); 1712 PME de 1991 à 1995 (Boussaâ, 2000);2551 PME de 1994 à 2001 (Ziane, 2004);1898 PME de 1995 à 2002 (Trabelsi, 2006); 1535 PME de 2000 à 2007 (Dufour et Molay, 2010). L'étude des 56605 TPE de 1998 à 2006 (Bellettre, 2010) fait exception ( $c f$. annexe IV). Cependant, cette base est plus restreinte que celle de la Banque de France (base FIBEN). 
Ensuite, les entreprises ont été sélectionnées selon leur appartenance sectorielle en utilisant la Nomenclature d'activité française (NAF), niveau 60 de l'INSEE, et qui est résumée en huit indicateurs sectoriels ( $c f$. annexe II).

Les entreprises retenues dans notre échantillon sont celles dont les données financières sont mises à jour et disponibles sur notre période d'observation de cinq ans (2000-2004)5. Leurs comptes sont disponibles et ont été publiés durant six années consécutives au 31 décembre de l'année, correspondant à 12 mois d'activité.

Les entreprises retenues sont celles qui ont une durée d'activité (âge) supérieure à cinq ans. Nous avons choisi d'exclure les jeunes entreprises ou celles nouvellement créées dont le régime fiscal et les besoins de financement sont particuliers ou dont la publication des comptes est lacunaire. Nous obtenons ainsi un panel cylindré de PME qui nous permet de disposer d'un nombre suffisant d'observations pour explorer la dimension temporelle dans l'analyse économétrique.

À l'issue de la sélection, des opérations de nettoyage ont supprimé les observations présentant des valeurs incohérentes. Le tableau 2 résume les étapes de la sélection de l'échantillon et montre que le critère de mise à jour conduit à supprimer un grand nombre d'entreprises, car cette base ne fait pas l'objet d'une mise à jour régulière.

TABLEAU 2

Résumé des étapes de sélection de l'échantillon

\begin{tabular}{lcc}
\hline Critères de choix & Nombre initial & Nombre restant \\
\hline Effectif moyen $<250$ & 209936 & 154570 \\
Appartenance sectorielle (NAF) & 154570 & 151871 \\
Entreprises mises à jour & 151871 & 3170 \\
Comptes disponibles & 3170 & 2111 \\
Clôture des comptes au 31 décembre & 2111 & 1621 \\
$\begin{array}{l}\text { Date de création antérieure } \\
\text { au 1999-12-31 }\end{array}$ & 1621 & 1581 \\
Nettoyage & 1581 & 1520 \\
\hline
\end{tabular}

Source: DIANE, nos calculs.

5. La base de données DIANE fournit des informations sur une période maximale des dix dernières années. 


\subsection{Statistiques descriptives}

La répartition de notre échantillon par taille selon le type des PME n'est pas proportionnelle aux données de l'OSEO sur le plan national (Savajol, 2003), soit respectivement $92,85 \%, 6 \%$ et $1 \%$ pour les microentreprises, les petites et moyennes entreprises ( $c f$. annexe III). D'après le tableau 3 , notre panel présente une structure équilibrée, soit respectivement $44,01 \%, 42,30 \%$ et $13,68 \%$ pour les microentrerprises, les petites et les moyennes entreprises. La structure cylindrée de notre panel (nous avons choisi de ne retenir que les entreprises disposant de toutes les données sur l'ensemble de la période concernée) et la méthodologie de sélection expliquent cette divergence.

TABLEAU 3

Répartition par taille selon le type des entreprises (année 2004)

\begin{tabular}{lcc}
\hline Taille & Fréquence & $\mathbf{( \% )}$ \\
\hline Microentreprises (-10 employés) & 669 & 44,01 \\
Petites entreprises (10-49 employés) & 643 & 42,30 \\
Moyennes entreprises (50-249 employés) & 208 & 13,68 \\
\hline Total & 1520 & 100,00 \\
\hline
\end{tabular}

Source: DIANE, nos calculs.

Le tableau 4 montre la répartition de notre panel par secteur selon la Nomenclature d'activité française (NAF), qui regroupe les branches de l'industrie ( $c f$. annexe II), afin de se rapprocher de la méthode adoptée par l'INSEE en ce qui concerne la ventilation des secteurs ${ }^{6}$ selon les trois catégories de PME. L'ensemble des secteurs est représenté dans le panel à l'exception de l'agriculture, des activités financières et de l'administration, qui relèvent d'un mode de financement différent. Les secteurs de l'industrie, du commerce, de la construction et des services représentent respectivement $20,20 \%, 11,05 \%, 35,46 \%$ et $33,29 \%$. Les secteurs du commerce et des services concentrent le plus grand nombre des entreprises de notre panel. Les microentreprises représentent respectivement $17,24 \%$ et $15,53 \%$ pour les

6. Définis par l'INSEE comme l'ensemble des activités économiques (y compris libérales) des établissements de l'industrie, du commerce et des services. Les activités relatives à l'agriculture et les activités financières ainsi que les associations, les «holdings» et les sociétés civiles immobilières (sans salariés) sont exclues (MEF, 2002). 
secteurs des services et du commerce. Les petites entreprises se concentrent plus dans le secteur du commerce avec $16,32 \%$. Les moyennes entreprises sont réparties également dans tous les secteurs.

TABLEAU 4

Répartition par taille selon le type de secteur d'activité (année 2004)

\begin{tabular}{|c|c|c|c|c|c|c|}
\hline \multirow[t]{2}{*}{ Taille } & & \multicolumn{4}{|c|}{ Secteur } & \multirow[t]{2}{*}{ Total } \\
\hline & & Industrie & Commerce & Construction & Services & \\
\hline \multirow{4}{*}{$\begin{array}{l}\text { Micro- } \\
\text { entreprises }\end{array}$} & $\mathrm{N}$ & 107 & 236 & 64 & 262 & 669 \\
\hline & $\%$ & 7,04 & 15,53 & 4,21 & 17,24 & 44,01 \\
\hline & $\%$ secteur & 15,99 & 35,28 & 9,57 & 39,16 & \\
\hline & $\%$ taille & 34,85 & 43,78 & 38,10 & 51,78 & \\
\hline \multirow{4}{*}{$\begin{array}{l}\text { Petites } \\
\text { entreprises }\end{array}$} & $\mathrm{N}$ & 143 & 248 & 82 & 170 & 643 \\
\hline & $\%$ & 9,41 & 16,32 & 5,39 & 11,18 & 42,30 \\
\hline & $\%$ secteur & 22,24 & 38,57 & 12,75 & 26,44 & \\
\hline & $\%$ taille & 46,58 & 46,01 & 48,81 & 33,60 & \\
\hline \multirow{4}{*}{$\begin{array}{l}\text { Moyennes } \\
\text { entreprises }\end{array}$} & $\mathrm{N}$ & 57 & 55 & 22 & 74 & 208 \\
\hline & $\%$ & 3,75 & 3,62 & 1,45 & 4,87 & 13,68 \\
\hline & $\%$ secteur & 27,40 & 26,44 & 10,58 & 35,58 & \\
\hline & $\%$ taille & 18,57 & 10,20 & 13,10 & 14,62 & \\
\hline \multirow{2}{*}{ Total } & & 307 & 539 & 168 & 506 & 1520 \\
\hline & & 20,20 & 35,46 & 11,05 & 33,29 & 100,00 \\
\hline
\end{tabular}

Source: DIANE, nos calculs.

En complément de l'analyse de la structure sectorielle de l'échantillon, nous détaillons les principales caractéristiques financières de notre panel. Le tableau 5 et la figure 1 ci-après synthétisent, respectivement, l'information relative aux choix des variables utilisées dans notre modèle selon la taille de l'entreprise, et ce, pour la dernière année 2004, ainsi que l'évolution du taux moyen d'endettement des PME du panel sur l'ensemble de la période. 
TABLEAU 5

Statistiques descriptives des variables financières selon le type de PME (2004)

\begin{tabular}{|c|c|c|c|c|c|}
\hline Taille & $\begin{array}{l}\text { Types de } \\
\text { variables }\end{array}$ & Variables & $\mathbf{N}$ & Moyenne & Écart type \\
\hline \multirow{6}{*}{$\begin{array}{l}\text { Micro- } \\
\text { entreprises }\end{array}$} & $\begin{array}{l}\text { Variable } \\
\text { expliquée }\end{array}$ & Taux d'endettement & 669 & 0,6360 & 0,3469 \\
\hline & \multirow{5}{*}{$\begin{array}{l}\text { Variables } \\
\text { explicatives }\end{array}$} & Âge & 669 & 17,9791 & 14,6258 \\
\hline & & Taux d'investissement & 669 & 0,5538 & 2,5246 \\
\hline & & Crédit interentreprises & 669 & 0,0168 & 0,2364 \\
\hline & & Marge d'autofinancement & 669 & 0,3246 & 8,5133 \\
\hline & & Garanties & 669 & 0,2780 & 0,2524 \\
\hline \multirow{6}{*}{$\begin{array}{l}\text { Petites } \\
\text { entreprises }\end{array}$} & $\begin{array}{l}\text { Variable } \\
\text { expliquée }\end{array}$ & Taux d'endettement & 643 & 0,6557 & 0,2239 \\
\hline & \multirow{5}{*}{$\begin{array}{l}\text { Variables } \\
\text { explicatives }\end{array}$} & Âge & 643 & 20,8911 & 14,9301 \\
\hline & & Taux d'investissement & 643 & 0,3309 & 0,9605 \\
\hline & & Crédit interentreprises & 643 & 0,0151 & 0,2547 \\
\hline & & Marge d'autofinancement & 643 & 0,1133 & 0,3189 \\
\hline & & Garanties & 643 & 0,3266 & 0,2312 \\
\hline \multirow{6}{*}{$\begin{array}{l}\text { Moyennes } \\
\text { entreprises }\end{array}$} & $\begin{array}{l}\text { Variable } \\
\text { expliquée }\end{array}$ & Taux d'endettement & 208 & 0,7006 & 0,2266 \\
\hline & \multirow{5}{*}{$\begin{array}{l}\text { Variables } \\
\text { explicatives }\end{array}$} & Âge & 208 & 27,2019 & 19,4787 \\
\hline & & Taux d'investissement & 208 & 0,4464 & 1,2789 \\
\hline & & Crédit interentreprises & 208 & 0,0400 & 0,2465 \\
\hline & & Marge d'autofinancement & 208 & 0,1060 & 0,2137 \\
\hline & & Garanties & 208 & 0,3242 & 0,2226 \\
\hline
\end{tabular}

Source: DIANE, nos calculs. 
FIGURE 1

Évolution du taux d'endettement (2000-2004)

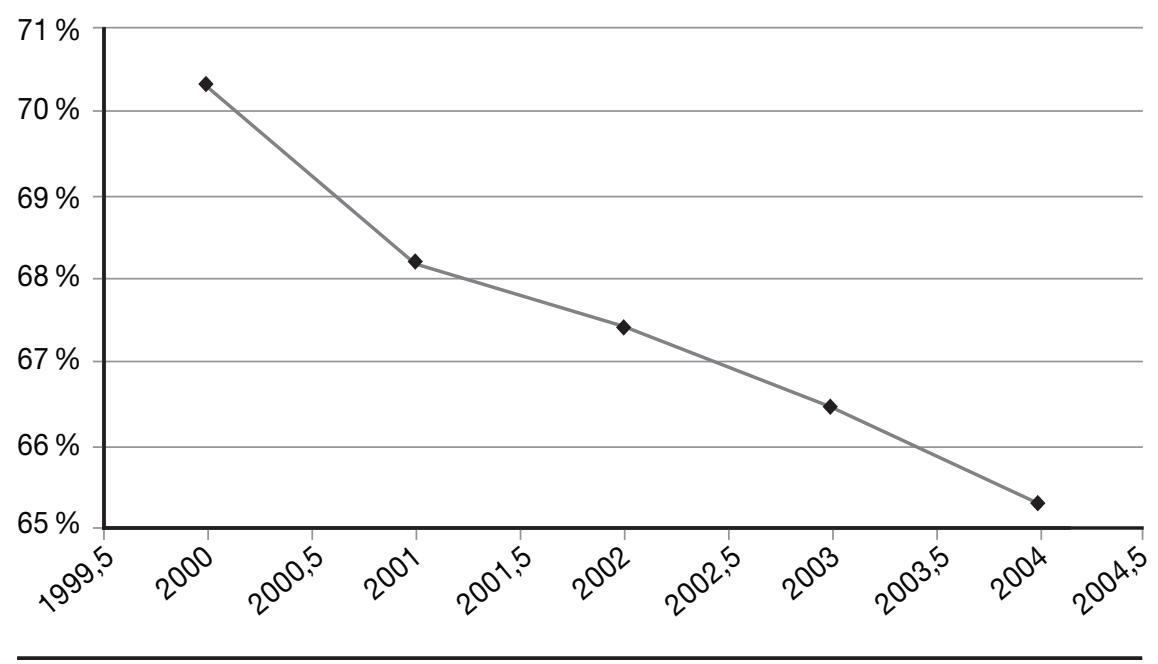

Source: DIANE.

Entre 2000 et 2001, les entreprises de notre panel ont enregistré un fort désendettement: le taux moyen d'endettement recule de 70,3\% à $68,1 \%$, soit près de 2,2 points. Sur le reste de la période, le désendettement se poursuit à un rythme ralenti: le taux moyen diminue de près de 0,8 point chaque année jusqu'à la fin de la période. Cette évolution peut être expliquée par l'éclatement de la bulle Internet, qui ouvre une phase de désendettement massif de la part des entreprises, et par la volonté des dirigeants d'entreprises de renforcer les fonds propres ${ }^{7}$.

Ce taux moyen d'endettement est une fonction croissante de la taille avec $64 \%, 66 \%$ et $70 \%$ respectivement pour les microentreprises, les petites entreprises et les moyennes entreprises; ce résultat peut être expliqué par le fait que les entreprises de petite taille préfèrent l'autofinancement au financement par la dette. La dispersion de cette variable est plus marquée pour les microentreprises que pour les autres catégories.

7. L'étude de Ziane (2004), sur une période antérieure, montre que le désendettement (1994-1998) fait place à l'endettement lors de la période de croissance (1998-2001). L'endettement est donc sensible à la conjoncture. 
Selon la Banque de France, ce désendettement correspond, du côté de la demande qui est prépondérante, à une baisse du taux d'investissement de 2000 à 2004 (Cayssials, Kremp et Peter, 2007), tandis que du côté de l'offre, le coût du crédit baisse également (tableau 6).

TABLEAU 6

\section{Coût moyen du crédit (2000-2004)}

\begin{tabular}{lccccc}
\hline & $\mathbf{2 0 0 0}$ & $\mathbf{2 0 0 1}$ & $\mathbf{2 0 0 2}$ & $\mathbf{2 0 0 3}$ & $\mathbf{2 0 0 4}$ \\
\hline Escompte & $6,39 \%$ & $5,27 \%$ & $5,03 \%$ & $4,13 \%$ & $3,82 \%$ \\
Découvert & $6,59 \%$ & $5,29 \%$ & $5,15 \%$ & $4,12 \%$ & $3,82 \%$ \\
$\begin{array}{l}\text { Autres } \\
\text { crédits CT }\end{array}$ & $5,53 \%$ & $4,62 \%$ & $4,04 \%$ & $3,32 \%$ & $3,10 \%$ \\
Crédits MLT & $5,91 \%$ & $5,29 \%$ & $4,77 \%$ & $4,10 \%$ & $3,44 \%$ \\
\hline
\end{tabular}

Source: Nos calculs d'après le Bulletin de la Banque de France.

Nous n'avons pas retenu la variable «coût du crédit». La relation inverse entre coût et montant unitaire du crédit est régulièrement relevée par les enquêtes de la Banque de France; elle va de pair, au sein de chaque tranche de montants, avec une relation également inverse entre coût du crédit et taille des entreprises. Les taux appliqués aux crédits nouveaux d'un montant inférieur à 250000 euros varient de $4,8 \%$ pour les microentreprises $(\mathrm{CA}<2 \mathrm{M} €)$ à 3,8\% pour les moyennes entreprises $(\mathrm{CA}<50 \mathrm{M} €)$.

Différentes études mettent en évidence un rationnement du crédit au moins au cours des années 1990. Le modèle de déséquilibre d'Aubier et Cherbonnier (2009) montre que les PME auraient éprouvé des difficultés d'accès au crédit bancaire à la fin des années 1990 et particulièrement en 2001, mais que ces contraintes se seraient desserrées depuis 2003. Du côté de l'offre, la faiblesse des taux d'intérêt, des conditions d'octroi des banques plus souples, des mesures favorables au financement bancaire des petites entreprises (notamment la suppression en 2003 du taux d'usure pour les entreprises, puis en 2005 pour les entrepreneurs individuels) et la création d'une banque publique du développement des PME (devenue OSEO en 2005) sont favorables à la croissance des crédits bancaires des petites et moyennes entreprises. Cependant, du côté de la demande, les PME augmentent leur trésorerie d'abord pour un motif de précaution à court terme (faire face à des chocs négatifs) plus que pour satisfaire un besoin de financer un 
investissement de moyen terme, ce qui peut expliquer à la fois la substitution de la trésorerie qui s'accroît aux crédits bancaires qui diminuent (Chertok et al., 2009).

\section{Méthodologie économétrique et résultats}

\subsection{Un modèle de panel cylindré}

Le modèle économétrique utilisé cherche à expliquer la variable taux d'endettement des firmes par différents indicateurs qui représentent les variables explicatives. La présence d'une perturbation dans le modèle est expliquée par la présence des autres indicateurs jugés moins pertinents et qui ne sont pas explicitement considérés. La présence de cette hétérogénéité des comportements ou de l'hétérogénéité inobservée pose des problèmes économétriques que la méthode des moindres carrés ordinaires (MCO) ne permet pas de résoudre. D'autres estimateurs ont été élaborés pour contrôler les effets sur les valeurs des paramètres estimés: Within (ou intra-individuel), Between (moyennes interindividuelles) et MCQG (moindres carrés quasi généralisés).

Les modèles à effets fixes (Within) et à effets aléatoires (MCQG) permettent, à la différence de la méthode des moindres carrés ordinaires (MCO), de prendre en compte l'hétérogénéité des données, mais les hypothèses sur la nature des effets spécifiques diffèrent d'un modèle à l'autre. Dans le premier modèle, les effets spécifiques sont, par hypothèse, corrélés avec les variables explicatives du modèle. Dans le second modèle, les effets spécifiques sont supposés indépendants des variables explicatives du modèle. Pour choisir entre ces deux modèles, Hausman (1978) propose un test de spécification qui utilise les différences de propriétés des deux estimateurs selon les deux cas: sous $H_{0}$, les deux estimateurs sont convergents, mais seul l'estimateur des MCQG est asymptotiquement efficace; sous $H_{1}$, seul l'estimateur du Within est convergent, mais il n'est pas asymptotiquement efficace.

Le modèle que nous testons par la suite est de la forme suivante ${ }^{8}$ :

8. Avec les notations économétriques usuelles, le modèle s'écrit ainsi: $Y_{i t}=\alpha+\beta X_{i t}+\mu_{i}+\varepsilon_{i t}$.

$\mu_{i}$ est un effet individuel, rendant compte de phénomènes non considérés dans le modèle, invariant dans le temps et qui exercent une influence sur la variable expliquée $Y$.

$\varepsilon_{i t}$ considère les effets non systématiques dans le temps d'autres facteurs, tels que des variables omises ainsi que des chocs exogènes.

$\mu_{i} \sim$ i. i.d $\left(0, \sigma_{\mu}^{2}\right)$ et $\varepsilon_{i t} \sim i . i . d\left(0, \sigma_{\varepsilon}^{2}\right)$ sont mutuellement indépendants, non autocorrélés et indépendants des variables explicatives $x$. 
$Y_{i t}=\alpha_{0}+\alpha_{1}$ dummymicro $_{i t}+\alpha_{2}$ dummymoyenne $_{i t}+\alpha_{3}$ dummycommerce $_{i t}$

$+\alpha_{4}$ dummyindustrie $_{i t}+\alpha_{5}$ dummyservice $_{i t}+\alpha_{6} A G E_{i t}+\alpha_{7} T I N V_{i t}+\alpha_{8} C_{1} E_{i t}$

$+\alpha_{9} M A F_{i t}+\alpha_{10} G A R_{i t}+\mu_{i}+\varepsilon_{i t}$

$\alpha_{0}$ : une constante qui peut être interprétée comme le taux d'endettement moyen des entreprises.

$Y_{i t}$ : la variable à expliquer est le taux d'endettement au sens large (TD).

Nombre d'entreprises $i=1, \ldots, 1520$

Nombre d'années $t=1, \ldots, 5$

Nous disposons de 7600 observations.

\subsection{Résultats empiriques et interprétations}

Les résultats de l'estimation du modèle à effets fixes (Within) et à effets aléatoires (MCQG) indiquent que les $\mathrm{R}^{2}$ sont relativement faibles. Pour le modèle à effets fixes, le $\mathrm{R}^{2}$ le plus pertinent est le $\mathrm{R}^{2}$ Within, car il indique la part de la variabilité intra-individuelle de la variable dépendante expliquée par celles des variables explicatives. Le test de Fisher et le test de Wald pour le modèle à effets fixes et le modèle à effets aléatoires sont respectivement significatifs à $1 \%$, ce qui montre que notre modèle est significatif. La probabilité du test de Breusch-Pagan montre que les effets aléatoires sont globalement significatifs au seuil de $1 \%$. La probabilité du test de Hausman Within comparée aux MCQG est significative au seuil de $1 \%$, ce qui implique que les effets spécifiques sont corrélés avec les variables explicatives et que le modèle à effets fixes est préférable au modèle à effets aléatoires. On peut donc considérer l'estimation du modèle à effets fixes (Within) comme une référence.

Mais l'estimation Within (ou intra-individuelle) ne nous permet pas d'estimer l'impact d'une variable invariante dans le temps (la variable secteur dans notre cas) parce que, dans ce modèle, les données sont transformées en différence par rapport à la moyenne individuelle. Afin de surmonter cette limite, il conviendrait d'utiliser Between (moyennes interindividuelles), mais ses résultats sont très proches de ceux des MCO. C'est la raison pour laquelle nous avons choisi un estimateur des variables instrumentales permettant d'estimer les paramètres d'un modèle qui comporte des variables constantes dans la dimension temporelle, et ainsi d'obtenir des estimations convergentes et efficientes lorsque les perturbations sont corrélées avec certaines variables explicatives du modèle. Les instruments utilisés sont les suivants: premièrement, les variables explicatives variant dans le temps, doublement exogènes, exprimées à la fois en moyennes individuelles et en écarts à la moyenne individuelle; deuxièmement, les variables explicatives invariantes dans le 
temps, doublement exogènes; enfin, les variables explicatives variant dans le temps, simplement exogènes, exprimées en écarts aux moyennes individuelles (Hausman et Taylor, 1981).

Puisque notre panel est cylindré, nous avons préféré la méthode des variables instrumentales d'Amemiya et MaCurdy (1986), qui utilisent des instruments additionnels pour améliorer l'efficacité des estimateurs. Les variables explicatives que nous supposons endogènes et qui varient dans le temps sont le taux d'investissement et la marge d'autofinancement ${ }^{9}$. L'utilisation des instruments est validée par le test de Sargan, ce qui signifie que les instruments utilisés ne sont pas corrélés avec les perturbations du modèle. Par la suite, nous commentons uniquement les résultats de l'estimateur d'Amemiya et MaCurdy (1986).

La variable qui reflète le choix entre capitaux propres et dette est le taux d'endettement total, au sens large sans distinction de nature et d'échéance. Le tableau 7 ci-dessous présente les résultats de l'estimation économétrique.

\section{TABLEAU 7}

Résultats des estimations

(variable expliquée : taux d'endettement total)

\begin{tabular}{lcccc}
\hline Variables & MCO & Within & MCQG & Ama-MaCurdy \\
\hline Dummy micro & $-0,013$ & $0,016^{*}$ & 0,008 & $0,013^{*}$ \\
& $(-1,609)$ & $(1,779)$ & $(1,0303)$ & $(1,6786)$ \\
\hline Dummy moyenne & $0,059^{* * *}$ & $-0,027^{*}$ & 0,008 & $-0,013$ \\
& $(6,711)$ & $(-1,683)$ & $(0,6058)$ & $(-0,9228)$ \\
\hline Dummy commerce & $-0,114^{* * *}$ & - & $-0,128^{* * *}$ & $-0,133^{* * * *}$ \\
& $(-6,592)$ & - & $(-5,3681)$ & $(-3,4002)$ \\
\hline Dummy industrie & $-0,128^{* * *}$ & - & $-0,129^{* * *}$ & $-0,130^{* * * *}$ \\
& $(-8,791)$ & - & $(-5,0914)$ & $(-3,0752)$ \\
\hline
\end{tabular}

9. Les variables exogènes variant dans le temps sont: dummy micro, dummy moyenne, âge, crédit interentreprises, et garanties; les variables exogènes invariantes sont les secteurs: dummy commerce, dummy industrie et dummy services. Seule l'hypothèse d'exogénéité stricte des variables explicatives permet l'introduction d'instruments supplémentaires. Ces variables sont considérées comme exogènes parce qu'elles semblent ne pas être corrélées avec une caractéristique inobservable et propre aux PME, non prise en compte dans le modèle. 


\begin{tabular}{lcccc}
\hline Dummy services & $\begin{array}{c}-0,086^{* * *} \\
(-5,296)\end{array}$ & - & $\begin{array}{c}-0,093^{* * *} \\
(-3,9822)\end{array}$ & $\begin{array}{c}-0,095^{* *} \\
(-2,4377)\end{array}$ \\
\hline Âge (AGE) & $-0,003^{* * *}$ & 0,023 & $-0,002^{* * *}$ & $-0,002^{* * *}$ \\
& $(-10,899)$ & $(1,497)$ & $(-5,2376)$ & $(-2,9126)$ \\
\hline Taux & $-0,002^{* * *}$ & 0,001 & 0,000 & $0,001^{*}$ \\
d'investissement & $(-2,774)$ & $(1,608)$ & $(0,7799)$ & $(1,6805)$ \\
(TINV) & $-0,297^{* * *}$ & $-0,383^{* * *}$ & $-0,352^{* * *}$ & $-0,371^{* * *}$ \\
\hline Crédit & $(-7,805)$ & $(-18,416)$ & $(-19,8171)$ & $(-20,2938)$ \\
interentreprises & 0,000 & $-0,000$ & $-0,000$ & $-0,000$ \\
(CIE) & $(1,589)$ & $(-0,759)$ & $(-0,3326)$ & $(-0,7876)$ \\
\hline Marge d'auto- & $0,082^{* * *}$ & 0,049 & $0,061^{* * *}$ & $0,054^{* * *}$ \\
financement (MAF) & $(3,372)$ & $(1,471)$ & $(2,5761)$ & $(1,9967)$ \\
\hline Garanties (GAR) & $0,806^{* * * *}$ & 0,199 & $0,809^{* * *}$ & $0,810^{* * *}$ \\
\hline Constante & $(39,669)$ & $(0,641)$ & $(35,1233)$ & $(21,7810)$ \\
\hline Observations & 7600 & 7600 & 7600 & 7600 \\
\hline R 2 & 0,090 & 0,058 & 0,094 & \\
\hline Test de Fisher & & 53,67 & & \\
Prob > F & 0,0000 & & \\
\hline Test Haun & & & & \\
\hline
\end{tabular}

Test Hausman

Within comparé

$\begin{array}{lcc}\text { à MCQG } & 57,49 & 56,67 \\ \text { Prob Hausman } & 0,0000 & 0,0000\end{array}$

Test de Sargan

30,844

$\mathrm{P}$-value

0,1267

Wald de chi $^{2}$

516,7

Prob $>\mathrm{chi}^{2}$

0,0000

Test de Breusch-

Pagan

5995,86

Prob $>$ chi $^{2}$

0,0000

* : Significatif au seuil de 10\%:(t-statistics)

**: : Significatif au seuil de $5 \%$

*** : Significatif au seuil de $1 \%$.

Nous observons que l'effet de taille (effectif salarié) est présent dans notre étude. Le coefficient associé à la variable indicatrice dummy micro est positif et significatif au seuil de $10 \%$ et le coefficient associé à la 
variable indicatrice dummy moyenne est négatif, mais non significatif. La variable indicatrice dummy petite (effectif de 10-49 salariés) est considérée comme une référence. Ces résultats signifient que le taux d'endettement des entreprises de taille petite et moyenne est similaire. En revanche, le taux d'endettement des microentreprises est supérieur à celui des petites entreprises $(+1,3 \%)$. Ces résultats signifient que le taux d'endettement n'est pas une fonction croissante de la taille. L'hypothèse H1 d'une corrélation positive n'est donc pas vérifiée. L'effet de taille est ambigu (Rajan et Zingales, 1995); la taille peut être un indicateur d'informations pour les investisseurs externes, ce qui concerne tant l'importance des fonds propres que celle de la dette dans le total du bilan. Ce résultat infirme les prédictions de la théorie du compromis (TOT).

Notre résultat ne concorde pas avec ceux de Ziane (2004) et de Boussâa (2000), issus respectivement d'un échantillon de 2551 PME françaises et 1712 PME françaises, qui valident la théorie du financement hiérarchique selon laquelle les PME privilégient les sources de financement interne en raison des difficultés d'accès aux ressources externes auxquelles elles se heurtent. Ziane (2004) souligne que le découpage par taille valide les arguments de la théorie du compromis (coûts de faillite et d'agence) et peut justifier des comportements particuliers des microentreprises et des petites et moyennes entreprises.

La variable âge (AGE) de l'entreprise est significative au seuil de $1 \%$ et négativement corrélée avec la variable taux d'endettement (à peu près $-0,2 \%)$. L'hypothèse $\mathrm{H} 2$ d'une corrélation positive n'est pas validée. Ce résultat infirme les prédictions de la théorie du compromis (TOT), du fait que les entreprises plus âgées ont une meilleure réputation et une plus grande expérience qui peuvent réduire des coûts d'agence grâce à un signal positif. Il tend à confirmer les prédictions de la théorie du financement hiérarchique du fait que plus l'entreprise est âgée, plus sa capacité d'autofinancement augmente et moins elle est dans l'obligation de recourir au financement par dette. Cette relation négative entre l'âge de l'entreprise et son taux d'endettement est en concordance avec l'étude de Boussâa (2000), tandis que Ziane (2004) observe un effet ambigu.

Le secteur (SEC) est un facteur de différenciation importante. La variable indicatrice dummy construction est considérée comme une référence ${ }^{10}$. Les coefficients associés aux variables dummy commerce, dummy industrie et dummy services sont négatifs et significatifs. Ces résultats

$\overline{10 .}$ La construction est le secteur dont les investissements corporels dans la valeur ajoutée sont les plus faibles; ce secteur bénéficie également le plus des réductions d'impôts (Raspiller, 2007). 
confirment que ces trois secteurs sont moins endettés que le secteur de la construction, mais selon des pourcentages différents pour les secteurs des services $(-9,5 \%)$, de l'industrie $(-13 \%)$ et du commerce $(-13,3 \%)$. L'hypothèse $\mathrm{H} 3$ est vérifiée: le secteur d'activité exerce une influence sur le taux d'endettement de l'entreprise. Le secteur d'activité de l'entreprise, bien que mesuré à un niveau agrégé, peut refléter d'une manière synthétique le risque lié à l'activité de l'entreprise; il peut également refléter des différences de traitement fiscal ou d'information des créanciers par rapport aux perspectives de croissance (Bédué, 1997).

Le taux d'investissement (TINV) reflète les opportunités de croissance de l'entreprise; il mesure le degré d'asymétrie d'information et les conflits d'agence qui existent entre les dirigeants de l'entreprise et les bailleurs de fonds. Le signe de coefficient associé à cette variable est positif et significatif au seuil de $10 \%$; un taux d'investissement de $1 \%$ s'accompagne d'une augmentation du taux d'endettement d'environ $0,1 \%$. L'hypothèse $\mathrm{H} 4$ d'une liaison positive entre le taux d'investissement et le taux d'endettement est donc validée. Ce lien positif et significatif confirme les prédictions de la théorie de financement hiérarchique (POT) et infirme celles de la théorie de l'agence (Myers, 1977). Si l'on considère la difficulté d'accès aux marchés financiers pour l'obtention d'apports sous forme de fonds propres et la volonté des propriétaires-dirigeants de conserver l'autonomie et l'indépendance de leurs entreprises, il apparaît cohérent que les PME qui ont des opportunités de croissance s'endettent en ayant recours au crédit bancaire. Ce lien positif est également relevé dans d'autres études sur les PME françaises (Ziane, 2004; Trabelsi, 2006).

Le coefficient associé à la variable crédit interentreprises (CIE) est négatif et significatif à $1 \%$. Une augmentation du solde du crédit interentreprises réduit d'environ $37 \%$ le taux d'endettement. L'hypothèse $\mathrm{H} 5$, selon laquelle les entreprises utilisant plus de crédit interentreprises ont un taux d'endettement moins élevé, est validée. Ce lien négatif et significatif entre ces deux variables confirme les prédictions de la théorie du financement hiérarchique (POT) et la thèse défendue par Petersen et Rajan (1994), qui considèrent que la dette commerciale est un substitut à la dette financière ${ }^{11}$. En revanche, Trabelsi (2006) constate une relation positive et significative entre ces deux variables, laquelle souligne le rôle des effets de réputation de la PME auprès de ses partenaires commerciaux dans son accès aux crédits bancaires.

11. Carbó-Valverde, Rodríguez-Fernández et Udell (2009) corroborent une version élargie de la POT en distinguant les PME non rationnées qui ont recours au crédit bancaire et celles qui recourent au crédit interentreprises lorsqu'elles sont rationnées. 
La variable des garanties offertes (GAR) est significative et positivement corrélée avec le taux d'endettement (TD). L'hypothèse H6 est donc validée. Les entreprises ayant des immobilisations corporelles et des stocks dans le bilan sont plus endettées que les autres $(+5,4 \%)$. Le collatéral joue un rôle important dans la relation de crédit (Lopez-Gracia et SogorbMira, 2008); il permet de réduire les coûts d'agence et incite davantage les créanciers à s'engager dans des prêts rémunérés d'échéance longue (Jensen et Meckling, 1976; Harris et Raviv, 1990). Ce lien positif observé entre l'endettement et les garanties confirme donc les prédictions de la théorie du compromis (TOT). En raison de l'importance des coûts de défaillance et des coûts d'agence associés aux prêts à destination des PME, les bailleurs de fonds exigent des garanties comme critère de sélection obligatoire pour l'octroi de prêt. Notre résultat concorde avec ceux de Ziane (2004) et de Trabelsi (2006).

La marge d'autofinancement (MAF), qui est un indicateur des ressources de financement interne, a une influence négative sur le taux d'endettement, mais cet impact n'est pas significatif dans notre étude. Contrairement à ce qui était attendu, la marge d'autofinancement n'a pas d'effet sur le taux d'endettement et nous ne pouvons pas valider ou invalider l'hypothèse $\mathrm{H} 7$ d'une corrélation négative entre ces deux variables. Nous ne pouvons donc pas confirmer ou infirmer les prédictions de la théorie du financement hiérarchique (POT).

\section{Conclusion}

Nous avons tenté d'expliquer les déterminants du taux d'endettement des PME en France sur un large échantillon de 1520 PME observées sur une période de cinq ans. Notre objectif était de tester la pertinence des hypothèses de la théorie du compromis (TOT) et de la théorie du financement hiérarchique (POT), visant à expliquer la structure financière des grandes entreprises cotées, appliquées aux choix de financement et à la spécificité financière des PME.

Il n'existe pas une théorie universelle des déterminants de la structure financière des entreprises en général et des PME en particulier; chaque théorie dépend des conditions économiques et des caractéristiques financières de la firme. Les effets des variables explicatives choisies diffèrent selon la théorie choisie. Selon la théorie du financement hiérarchique (POT), il devrait y avoir une relation négative entre l'âge de l'entreprise et le levier financier; a contrario, selon la théorie du compromis (TOT), il devrait y avoir une corrélation positive entre ces deux variables. Cependant, nos résultats ne permettent pas toujours de valider ou d'invalider l'une ou l'autre théorie, lesquelles ne s'opposent pas en tout point. 
Premièrement, l'effet de taille est observé dans notre étude, mais il s'avère ambigu. La taille peut, donc, être un indicateur d'informations pour les investisseurs externes, ce qui concerne l'importance tant des fonds propres que celle de la dette dans le total du bilan; la théorie du compromis (TOT) est rejetée.

Deuxièmement, la relation négative de l'âge de l'entreprise avec le taux d'endettement infirme les prédictions de la théorie du compromis (TOT), selon lesquelles les entreprises plus âgées ont une meilleure réputation et une plus grande expérience qui peuvent conduire à la réduction des coûts d'agence grâce à un signal positif, donc à une augmentation de leur taux d'endettement.

Troisièmement, le secteur d'activité exerce une influence sur le taux d'endettement de l'entreprise. Malheureusement, nous ne pouvons pas déterminer la nature de cette influence parce que nous ne disposons pas des indicateurs de risque associés à chaque secteur d'activité.

Quatrièmement, la relation positive entre le taux d'investissement et le taux d'endettement confirme l'existence d'asymétries d'information et de conflits d'agence entre les dirigeants de l'entreprise et leurs créanciers. Ce résultat confirme les prédictions de la théorie de financement hiérarchique (POT) et infirme la théorie de l'agence (Myers, 1977).

Cinquièmement, le crédit interentreprises est négativement corrélé avec le taux d'endettement. Ce lien négatif et significatif entre ces deux variables confirme les prédictions de la théorie du financement hiérarchique (POT) et la thèse défendue par Petersen et Rajan (1994), qui considèrent que la dette commerciale est un substitut à la dette financière.

Enfin, nos résultats confirment la prédiction de la théorie du compromis (TOT) selon laquelle les entreprises qui disposent de garanties sont plus endettées que les autres.

Notre étude comporte des limites brièvement recensées ci-après qui suggèrent des prolongements de cette recherche. On peut identifier des problèmes liés à l'agrégation des informations comptables et donc à la mesure de certaines variables au sein de la base de données; dans une recherche ultérieure, nous aurons recours à une base de données complémentaire (ALISSE, INSEE). La période de cinq ans n'est pas suffisante pour obtenir des estimations convergentes des effets individuels; notre recherche couvrira l'ensemble de la période 2000-2009 et prendra en considération le rationnement du crédit. 
Les PME recouvrent des caractéristiques variées, notamment selon la taille et l'âge, le statut juridique ainsi que la propriété du capital. Ces deux derniers déterminants des comportements financiers méritent une exploration approfondie. Il semble difficile de concevoir un cadre théorique unifiant les comportements financiers, notamment pour les plus petites entreprises.

ANNEXE I

Les seuils relatifs aux PME, valables à partir du $1^{\text {er janvier } 2005}$

\begin{tabular}{lccc}
\hline Catégorie d'entreprise & Effectif & Chiffre d'affaires & Total du bilan \\
\hline Moyenne entreprise & $<250$ & $50 \mathrm{M} €$ & $43 \mathrm{M} €$ \\
Petite entreprise & $<50$ & $10 \mathrm{M} €$ & $10 \mathrm{M} €$ \\
Microentreprise & $<10$ & $2 \mathrm{M} €$ & $2 \mathrm{M} €$ \\
\hline
\end{tabular}

Source: Commission européenne.

\section{ANNEXE II \\ Classification des secteurs selon la nomenclature d'activité française (NAF)}

\begin{tabular}{lll}
\hline Numéro & Nom de l'activité & Codes activités \\
\hline 1 & $\begin{array}{l}\text { Industrie agricole et alimentaire (IAA), } \\
\text { énergie }\end{array}$ & 10 à 16, 23, 40, 41 \\
& Industrie des biens de consommation & 17 à 19, 35 à 37 \\
3 & Industrie automobile & 34 \\
4 & Industrie des biens d'équipement & 29 à 33 \\
5 & Industrie des biens intermédiaires & 20 à 22, 24 à 28 \\
6 & Construction & 45 \\
7 & Commerce & 50 à 52 \\
8 & $\begin{array}{l}\text { Service (transport, activités immobilières, } \\
\text { services aux entreprises, services aux }\end{array}$ & 60 à 62,63 à 64,70 à 71, \\
& particuliers, éducation, santé et action sociale) & 80 à 85 \\
\hline
\end{tabular}

Étant donné qu'elles fonctionnent avec un mode de financement différent, les activités agricoles, sylviculture et pêche (01 à 05), les activités financières (65 à 67), l'administration publique et les activités associatives et extra-associatives $(75,91,99)$ ont été exclues. 
ANNEXE III

Répartition selon la taille des PME dans l'économie française

\begin{tabular}{lcc}
\hline Nombre de salariés & Nombre d'entreprises & \% du total \\
\hline 0 (microentreprise) & 1215724 & 49,75 \\
1 à 9 (microentreprise) & 1053043 & 43,10 \\
10 à 49 (petite entreprise) & 146702 & 6,00 \\
50 à 199 (moyenne entreprise) & 21670 & 0,89 \\
200 à 249 (moyenne entreprise) & 1454 & 0,08 \\
Total PME & 2438593 & 99,80 \\
250 et plus & 4769 & 0,20 \\
\hline Total & 2443362 & 100,00 \\
\hline
\end{tabular}

Source: Savajol (2003).

ANNEXE IV

Panorama des travaux théoriques et empiriques sur les PME

\begin{tabular}{|c|c|c|c|}
\hline $\begin{array}{c}\text { Travaux } \\
\text { de référence }\end{array}$ & \multicolumn{3}{|c|}{$\begin{array}{c}\text { Travaux empiriques sur les PME } \\
\text { en France et en Europe }\end{array}$} \\
\hline $\begin{array}{l}\text { Auteurs } \\
\text { et année }\end{array}$ & $\begin{array}{l}\text { Auteurs } \\
\text { et année }\end{array}$ & $\begin{array}{l}\text { Échantillon, } \\
\text { pays et période }\end{array}$ & Résultats \\
\hline Ang (1991) & $\begin{array}{l}\text { Bartholdy et } \\
\text { Mateus (2005) }\end{array}$ & $\begin{array}{l}\text { Panel non cylindré } \\
\text { de } 998 \text { PME } \\
\text { (Portugal), } \\
\text { 1990-2000. }\end{array}$ & $\begin{array}{l}\text { Impact de la déductibilité } \\
\text { des charges d'intérêt sur } \\
\text { la structure financière } \\
\text { des PME non cotées. }\end{array}$ \\
\hline $\begin{array}{l}\text { Barnea } \\
\text { Haugen } \\
\text { et Senbet } \\
(1981 \mathrm{a} \\
1981 \mathrm{~b})\end{array}$ & $\begin{array}{l}\text { Bédué } \\
\text { (1997) }\end{array}$ & $\begin{array}{l}\text { Panel cylindré } \\
\text { de } 1387 \text { entreprises } \\
\text { (France), } \\
\text { 1989-1993. }\end{array}$ & $\begin{array}{c}\text { Valide la POT } \\
\text { Vérifie la théorie } \\
\text { de l'agence. } \\
\text { Met l'accent sur le } \\
\text { rôle des garanties dans } \\
\text { la relation banque- } \\
\text { entreprise. }\end{array}$ \\
\hline $\begin{array}{l}\text { Cornell et } \\
\text { Shapiro } \\
(1987)\end{array}$ & $\begin{array}{l}\text { Bellettre } \\
(2010)\end{array}$ & $\begin{array}{c}\text { Panel non cylindré } \\
\text { de 56605 TPE } \\
\text { (France), } \\
\text { 1998-2006. }\end{array}$ & $\begin{array}{l}\text { La POT n'explique pas la } \\
\text { préférence des TPE pour } \\
\text { les dettes financières, } \\
\text { par rapport aux comptes } \\
\text { courants d'associés. }\end{array}$ \\
\hline
\end{tabular}




\begin{tabular}{|c|c|c|c|}
\hline $\begin{array}{l}\text { Holmes et } \\
\text { Kent } \\
(1991)\end{array}$ & $\begin{array}{c}\text { Boussâa } \\
\text { (2000) }\end{array}$ & $\begin{array}{l}\text { Panel cylindré } \\
\text { de } 1712 \text { PME } \\
\text { (France), } \\
\text { 1991-1995. }\end{array}$ & $\begin{array}{l}\text { La POT et la théorie du } \\
\text { signal peuvent expliquer } \\
\text { la structure financière } \\
\text { des PME. Le crédit } \\
\text { interentreprises réduit } \\
\text { l'asymétrie d'information. }\end{array}$ \\
\hline $\begin{array}{l}\text { Pettit et Singer } \\
\text { (1985) }\end{array}$ & $\begin{array}{l}\text { Daskalakis } \\
\text { et Psillaki } \\
(2007)\end{array}$ & $\begin{array}{c}\text { Panel cylindré } \\
\text { de } 320 \text { PME (Italie), } \\
52 \text { entreprises } \\
\text { (Portugal), } 1252 \\
\text { entreprises (Grèce) et } \\
\text { 2006 PME (France), } \\
\text { 1997-2002. }\end{array}$ & Valident la POT. \\
\hline $\begin{array}{l}\text { Psillaki } \\
\text { (1995) }\end{array}$ & $\begin{array}{l}\text { Dufour } \\
\text { et Molay } \\
(2010)\end{array}$ & $\begin{array}{c}\text { Panel cylindré } \\
\text { de } 1535 \text { PME, France, } \\
\text { 2000-2007. }\end{array}$ & Valident la TOT. \\
\hline $\begin{array}{l}\text { Schnabel } \\
(1992)\end{array}$ & $\begin{array}{l}\text { Sogorb-Mira } \\
(2005)\end{array}$ & $\begin{array}{l}\text { Panel cylindré } \\
\text { de } 6482 \text { PME } \\
\text { (Espagne), } \\
\text { 1994-1998. }\end{array}$ & Valide la POT. \\
\hline \multirow[t]{2}{*}{$\begin{array}{l}\text { St-Pierre } \\
(1999)\end{array}$} & $\begin{array}{l}\text { Trabelsi } \\
(2006)\end{array}$ & $\begin{array}{c}\text { Panel cylindré } \\
\text { de 1898 PME } \\
\text { (France), 1995-2002. }\end{array}$ & $\begin{array}{l}\text { La POT et la TOT } \\
\text { sont compatibles. }\end{array}$ \\
\hline & $\begin{array}{l}\text { Ziane } \\
(2004)\end{array}$ & $\begin{array}{c}\text { Panel cylindré } \\
\text { de 2551 PME } \\
\text { (France), 1994-2001. }\end{array}$ & $\begin{array}{c}\text { La POT est plus utilisée, } \\
\text { mais sans exclure } \\
\text { la TOT. }\end{array}$ \\
\hline
\end{tabular}

Source: Composé par nos soins.

\section{Bibliographie}

ADAM, M.C., A. FARBER et P. MICHEL (1989), «Théorie financière et PME», Nouvelles de la Science et des Technologies, vol. 7, n 3, p. 59-64.

AMEMIYA, T. et T.-E. MACURDY (1986), «Instrumental-variable estimation of an error-components model», Econometrica, vol. 54, n 4, p. 869-880.

ANG, J. S. (1991), «Small business uniqueness and the theory of financial management», Journal of Small Business Finance, vol. 1, n 1, p. 1-13.

ANG, J.S. (1992), «On the theory of finance for privately held firms », Journal of Small Business Finance, vol. 1, n 3, p. 185-203. 
AUbIER, M. et F. CHERBONNIER (2009), «Les banques et le financement des PME en France», dans G. Chertok, P.-A. Malleray et P. Pouletty (dir.), Le financement des PME, Paris, La Documentation française, p. 127-142.

BALLING, M., B. BERNET et E. GNAN (2009), Financing SMEs in Europe, Société universitaire européenne de recherches financières (SUERF), Vienne.

BARNEA, A., R.A.HAUGEN et L.W.SENBET (1981a), «Market imperfections, agency problems and capital structure: a review», Financial Management, vol. 10, no 3, p. 7-22.

BARNEA, A., R.A. HAUGEN et L.W. SENBET (1981b), «An equilibrium analysis of debt financing under costly tax arbitrage and agency problems », Journal of Finance, vol. 36, p. 569-582.

BARTHOLDY, J. et C. MATEUS (2005), «Debt and taxes: evidence from bank-financed small and medium-sized firms », $12^{\text {th }}$ Annual Conference of the Multinational Finance Society, Athènes, Grèce.

BÉDUÉ, A. (1997), Choix financiers des entreprises et comportement bancaire, thèse, Université Paris X.

Belletante, B., N. LeVRatTo et B. PARANQUe (2001), Diversité économique et mode de financement des PME, Paris, L'Harmattan.

BelletTre, I. (2010), Les choix de financement des Très Petites Entreprises, thèse, Université Lille II.

BERGER, A. et G.F. UDELL (1990), «Collateral, loan quality, and bank risk », Journal of Monetary Economics, vol. 25, p. 21-42.

BESANKO, D. et A.V. THAKOR (1987), «Collateral and rationing: sorting equilibria in monopolistic and competitive market», International Economic Review, vol. $28, \mathrm{n}^{\circ} 3$, p. 671-689.

BESTER H. (1985), «Screening versus rationing in credit markets with imperfect information", American Economic Review, vol. 75, nº 4, p. 850-855.

BESTER H. (1987), «The role of collateral in credit markets with imperfect information», European Economic Review, vol. 31, no 4, p. 887-899.

BIAIS, B., P. HILLION et J.-F. MALECOT (1995), «La structure financière des entreprises: une investigation empirique sur données françaises », Économie et Prévision, vol. 120, n 120, p. 15-28.

Bourdieu, J. et B. COLIN-SEDILlOT (1993), «Structure de capital et coûts d'information: le cas des entreprises françaises à la fin des années quatre-vingt», Économie et Statistique, 268/269, p. 87-100.

BOUSSAA, N. (2000), Théorie financière contemporaine et PME: une analyse économique sur données de panel, thèse, Université Paris X.

BULAN, L. et Z. YAN (2010), «Firm maturity et pecking order theory», International Journal of Business et Economics, vol. 9, n 3, p. 179-200.

BRUNS, V. et M. FLETCHER (2008), «Banks' risk assessment of Swedish SMEs», Venture Capital, vol. 10, n 2, p. 171-94. 
CARBÓ-VAlVERdE, S., F. ROdRÍGUEZ-FERnÁNDEZ et G.F. UdELL (2009), «Bank market power et SME financing constraints », Review of Finance, vol. 13, n 2, p. 309-340.

CAYssials, J.-L., E. KREMP et C. PETER (2007), «Dix années de dynamique financière des PME en France», numéro spécial «Petites et Moyennes Entreprises », Bulletin mensuel de la Banque de France, no 165, septembre, p. 31-48.

Chertok, G., P. -A. De MAlleray et P. Pouletty (2009), Le financement des $P M E$, Rapport du Conseil d'analyse économique, Paris, La Documentation française.

ChitTenden, F., G. HAll et P. HutCHinson (1996), «Small firm growth, access to capital markets et financial structure: review of issues et an empirical investigation», Small Business Economics, vol. 8, p. 59-67.

COLOT, V. et P.-A. MiCHEL (1996), «Vers une théorie financière adaptée aux PME. Réflexion sur une science en genèse », Revue internationale PME, vol. $9, \mathrm{n}^{\circ} 1$, p. 143-166.

Coluzzi, C., A. Ferreto et C. Martinez-CARrascal (2009), «Financing obstacles et growth: an analysis for Euro area non-financial corporations », ECB Working Papers series, $\mathrm{n}^{\circ}$ 997, European Central Bank, janvier.

CORNELL, B. et A.C. SHAPIRO (1987), «Corporate stakeholders et corporate finance», Financial Management, vol. 16, $\mathrm{n}^{\circ}$ 1, p. 5-14.

DASKALAKIS, N. et M. PSILLAKI (2007), «Do country or firm factors explain capital structure? Evidence from SMEs in France et Greece», Applied Financial Economics, vol. 18, p. 87-97.

DELL'ARICCIA, G. et R. MARQUEZ (2004), «Information et bank credit allocation», Journal of Financial Economics, no 72 , vol. 1, p. 185-214.

DUFOUR, D. et É. MOLAY (2010), «La structure financière des PME françaises: une analyse sectorielle sur données de panel», XXXI ${ }^{\mathrm{e}}$ Congrès de l'Association francophone de comptabilité «Crises et nouvelles problématiques de la valeur», Nice, p. 1-18.

HARRIS, M. et A. RAVIV (1990), «Capital structure et the informational role of debt», Journal of Finance, vol. 45, n 2, p. 321-350.

HARRIS, M. et A. RAVIV (1991), «The theory of capital structure», Journal of Finance, vol. 46, n 1, p. 297-355.

HAUSMAN, J. A. (1978), «Specification tests in econometrics », Econometrica, vol. 46, p. 1251-1271.

HAUSMAN, J.A. et E. TAYLOR (1981), «Panel data and unobservable individual effects », Econometrica, vol. 49, p. 1377-1398.

HOLMES, S. et P. KENT (1991), «An empirical analysis of the financial structure of small and large Australian manufacturing enterprises », Journal of Small Business Finance, vol. 1, n 2, p. 141-154. 
JENSEN, M.C. et W. MECKLING (1976), «Theory of the firm: managerial behavior, agency costs and ownership structure», Journal of Financial Economics, vol. $3, n^{\circ} 4$, p. 305-360.

KREMP, E. et T. PHILLIPPON (2008), «Changing patterns of firm ownership et financing: evidence from SMEs in France», mimeo, Banque de France.

LELET, H. et D. PYLE (1977), «Informational asymmetric financial structure and financial intermediation », Journal of Finance, vol. 32, n 2, p. 371-387.

LEVRATTO, N. (1992), Une analyse du marché du crédit en termes de rationnement, thèse de sciences économiques, Université de Nice Sophia-Anipolis, Latapses.

LOPEZ-GRACIA, J. et F. SOGORB-MIRA (2008), «Testing trade-off and pecking order theories financing SMEs», Small Business Economics, vol. 31, p. 117-136.

MiLleR, M.H. (1977), «Debt and taxes », Journal of Finance, vol. 32, p. 261-275.

MINISTÈRE DE L'ÉCONOMIE ET DES FINANCES (2002), Les chiffres-clefs des PME, Études et Statistiques, Paris, MEF.

MOdigliani, F. et M.H. Miller (1958), «The cost of capital, corporation finance and the theory of investment», American Economic Review, vol. 48, n³ 3, p. 261-297.

Modigliani, F. et M.H. Miller (1963), «Corporate income taxes and the cost of capital: a correction», American Economic Review, vol. 53, n³ 3 p. 433-443.

MYERS, S.C. (1977), «Determinants of corporate borrowing», Journal of Financial Economics, vol. 5, p. 147-175.

MYERS, S.C. (2001), «Capital structure», The Journal of Economic Perspectives, vol. $15, \mathrm{n}^{\circ} 2$, p. $81-102$.

MYERS, S.C. et N.S. MAJLUF (1984), «Corporate financing and investment decisions when firms have information that investors do not have», Journal of Financial Economics, juin, vol. 13, n², p. 187-220.

OBSERVATOIRE EUROPÉEN DES PME (2001), «L'accès des entreprises au financement», document de travail, Commission européenne.

OBSERVATOIRE EUROPÉEN DES PME (2003), «L'accès au financement pour les $\mathrm{PME}$ », rapport $\mathrm{n}^{\circ}$ 2, Commission européenne.

PETERSEN, M.A. et R.G. RAJAN (1994), «The benefits of lending relationships: evidence from business data », Journal of Finance, vol. 49, n 1, p. 3-35.

PETTIT, R.R. et R.F. SINGER (1985), «Small business finance: a research agenda», Financial Management, vol. 14, no 3, p. 47-60.

PSILLAKI, M. (1995), «Rationnement du crédit et PME: une tentative de mise en relation », Revue internationale PME, vol. 8, nos 3-4, p. 67-90.

PSILLAKI, M. (1998), «Une présentation critique des mécanismes de révélation appliqués au marché du crédit», Revue française d'économie, vol. 13, nº 2, p. 29-58.

PSILlAKI, M., I.E. TSOLAS et D. MARGARITIS (2010), «Evaluation of credit risk based on firm performance», European Journal of Operational Research, vol. $201, \mathrm{n}^{\circ} 3$, p. $873-881$. 
RAJAN, R.G. et L. ZINGALES (1995), «What do we know about capital structure? Some evidence from international data », Journal of Finance, vol. 50, $\mathrm{n}^{\circ}$ 5, p. $1421-1460$.

RASPILLER, S. (2007), "Le taux réduit d'impôt sur les sociétés pour les PME», Trésor-Eco, $\mathrm{n}^{\circ} 23$, Direction générale du Trésor et de la politique économique (DGTPE).

Ross, S.A. (1977), «The determination of financial structure: the incentive-signalling approach », Bell Journal of Economics, vol. 8, nº 1, p. 23-40.

SAVAJOL, H. (2003), PME: clés de lecture (définitions, dénombrement, typologies), Les études de l'Observatoire des PME, Paris, OSEO.

SAVIGNAC, F. et P. SEVESTRE (2008), «Bank loans: do small and/or innovative firms face specific financial constraints?», mimeo, Banque de France.

SCHNABEL, J.A. (1992), «Small business capital structure choice », Journal of Small Business Finance, vol. 2, no 1, p. 13-21.

SOGORB-MIRA, F. (2005), «How SME uniqueness affects capital structure: evidence from a 1994-1998 Spanish data panel», Small Business Economics, vol. 25, n 5, p. 447-457.

STIGLITZ, J. (1969), «A re-examination of the Modigliani-Miller theorem», American Economic Review, vol. 59, n 5, p. 784-792.

STIGLITZ, J.E. et A. WEISS (1981), «Credit rationing in markets with imperfect information», American Economic Review, juin, vol. 71, n 3, p. 393-410.

STIGLITZ, J. E. et A. WEISS (1987), «Credit rationing with many borrowers », American Economic Review, vol. 77, p. 228-231.

ST-PIERRE, J. (1999), La gestion financière des PME. Théories et pratique, Sainte-Foy, Québec, Presses de l'Université du Québec.

TITMAN, S. et R. WESSELS (1988), «The determinants of capital structure choice», Journal of Finance, vol. 43, n 1, p. 1-19.

TRABELSI,A. (2006), Les déterminants de la structure du capital et les particularités $d u$ financement dans les PME: une étude sur données françaises, thèse, Université Paris IX Dauphine.

VANECKER, T. R. et S. MANIGART (2010), «Pecking order and debt capacity considerations for high growth companies seeking financing», Small Business Economics, 35, p. 53-69.

VASILIOU, D., N. ERIOTIS et N. DASKALAKIS (2009), «Testing the pecking order theory: the importance of methodology», Qualitative Research in Financial Markets, vol. 1, n 2, p. 85-96.

VOORDECKERS, W. et T. STEIJVERS (2006), «Business collateral et personal commitments in SME lending», Journal of Banking et Finance, vol. 30, p. 3067-3086.

ZIANE, Y. (2004), Structure financière, relations bancaires de long terme et financement interentreprises des PME françaises, thèse, Université Paris X. 